\title{
Quartet-metric general relativity: scalar graviton, dark matter, and dark energy
}

\author{
Yury F. Pirogov ${ }^{\mathrm{a}}$ \\ SRC Institute for High Energy Physics of NRC Kurchatov Institute, Protvino 142281, Russia
}

Received: 20 November 2015 / Accepted: 19 February 2016 / Published online: 18 April 2016

(C) The Author(s) 2016. This article is published with open access at Springerlink.com

\begin{abstract}
General relativity extended through a dynamical scalar quartet is proposed as a theory of the scalar-vectortensor gravity, generically describing the unified gravitational dark matter (DM) and dark energy (DE). The implementation in the weak-field limit of the Higgs mechanism for the extended gravity, with a redefinition of metric field, is exposed in a generally covariant form. Under a natural restriction on the parameters, the redefined theory possesses in the linearized approximation a residual transversediffeomorphism invariance, and consistently comprises the massless tensor graviton and a massive scalar one as a DM particle. The number of adjustable parameters in the full nonlinear theory and a partial decoupling of the latter from its weak-field limit noticeably extend the perspectives for the unified description of the gravity DM and DE in the various phenomena at the different scales.
\end{abstract}

\section{Introduction}

The unification of the superficially unrelated phenomena in nature seems to be the main trend in the contemporary fundamental physics. Among such the formally unrelated phenomena there are the so-called dark matter (DM) and dark energy (DE). While the former is concentrated mainly in galaxies and the cluster of galaxies, the latter is distributed all over the world, which results in their quite different manifestations. However, both DM and DE being extremely elusive, they may naturally have their common origin in a modification/extension of general relativity (GR). The latter is well known to be the generally covariant (GC) metric theory of gravity describing in vacuum one physical gravity mode, the massless two-component transverse-tensor graviton. It is commonly adopted that GR is not an ultimate theory of gravity. Nevertheless, it well may serve as a firm basis for a future (more) fundamental theory. In particular, among the

\footnotetext{
a e-mail: pirogov@ihep.ru
}

conceivable extensions to GR one may distinguish that with an additional (massive) scalar graviton. Such a GR extension, in comparison with a number of other ones, will mainly be addressed to in what follows. ${ }^{1}$

The masslessness of the tensor graviton is safely ensured in GR by the conventionally adopted general gauge invariance/relativity. At that, a scalar-gravity mode, contained a priori in the metric, gets unphysical as a by-product of the general gauge invariance/relativity. However, to ensure the generic property of the tensor masslessness it would suffice for a gravity theory to possess the invariance just under the volume-preserving/transverse diffeomorphisms (TDiff's) [2]. Given this, there could appear in metric one more physical gravity mode, the scalar one. A theory of gravity based on the explicit GR violation, with the residual TDiff invariance and an extra scalar mode contained in metric, was proposed in the non-GC form in [3-5] and further elaborated in [6]. In the explicitly GC form, such a theory was proposed in [7] and developed in [8-10]. At that, the explicit GR violation was treated as a raison d'être for the appearance of the gravitational DM. ${ }^{2}$ To such an interpretation, GC preservation proves to be crucial. The emergent scalargraviton DM proved to possess the generic properties conventionally assigned to DM. In particular, due to the coherent field of scalar gravitons there exists in vacuum a halo-type solution with a constant asymptotic rotation velocity $[9,10]$. Similarly to the black holes (BH's), serving as a signature for plain GR, the halo-type objects may serve as a signature for GR extended through the scalar graviton. Associated with the local scale invariance, the respective scalar graviton was called previously a systolon [10]. To ensure the GC preservation one should inevitably introduce a nondynamical scalar

\footnotetext{
${ }^{1}$ For a review on the extended theories of gravity, see, e.g., [1].

2 The term "GC violation", used previously in [7] and later, is more appropriately substituted here by the "GR violation" [10].
} 
density (of an unknown nature). ${ }^{3}$ This is the simplest theory realizing the scenario of the explicit GR violation with the gravity DM.

A more detailed study of the gravity DM may though require an extension of the scenario beyond the minimal one. Irrespective of DM, the general second-order effective Lagrangian with the explicit GR violation in the non-GC form was proposed in [11]. In the GC form, such a Lagrangian was obtained in the context of a nonlinear model in [12]. Similarly to the minimal case, to maintain GC under the explicit GR violation it is necessary to introduce a nondynamical affine connection expressed minimally through a quartet of the scalar fields. Such a proliferation of the uncontrollable nondynamical quantities in a semi-dynamical model makes one uneasy, both phenomenologically and theoretically, and may result in some conceptual problems. It would thus be desirable to make the effective field theory of gravity fully dynamical by converting the nondynamical quantities, minimally the scalar quartet, to the dynamical ones. A dynamical scalar quartet in the context of the space-time four-volume element was proposed originally in [14]. For an implementation of the Brout-Englert-Higgs (BEH) mechanism for the massive tensor gravity it was introduced in [15]. A special representation for the gravity Higgs fields in terms of the scalar quartet was proposed in [16] and worked out in [17]. It helps to solve some problems inherent to the massive modification of GR. Such a representation, with a modification hereof, is used for the consistent treatment of a more critical extension to GR in the present paper.

The paper develops the preceding results due to the author on the scalar-graviton/saystolon DM [7-10], modifying the semi-dynamical model to a fully dynamical theory. First of all, a general gravity kinetic Lagrangian, quadratic in the derivatives of the metric, in an explicitly GC form [12] is used instead of the minimal one. Besides, the explicit GR violation, with a nondynamical affine connection, is abandoned due to a dynamical scalar quartet. In Sect. 2, the full nonlinear theory of the quartet-metric (QM) GR, or, for short, the quartet-metric gravity (QMG) is considered. The GC frameworks for the unification of the gravity DM and DE are worked out. A coupled system of the classical field equations FE's for metric and the scalar quartet is presented. The minimal totally dynamical GR extension, in comfort with the previous semi-dynamical results $[9,10]$, is considered in more detail. In Sect. 3, the weak-field (WF) theory corresponding to the full nonlinear one is considered. The implementation of the BEH mechanism for the scalar-vector-tensor gravity

\footnotetext{
3 A nondynamical scalar density for a four-volume element appeared originally in the unimodular relativity (UR) [13]. Though inevitable in UR for GC, such a scalar density is nevertheless often missed, tacitly implying the special coordinates, as well as the absence of the scalardensity singularity (not necessarily fulfilled in a more general case).
}

in the arbitrary metric and quartet backgrounds is explicitly demonstrated in a GC form. The linearized approximation (LA) for the most general version of the theory, as well as for its natural reduction insuring TDiff invariance, is then considered. The latter case, being unitary and free of ghosts and the classical instabilities, is argued to consistently comprise the massless tensor graviton and a massive scalar one as a DM particle. The nearest and far-away prospects for QMG are shortly discussed in Conclusion.

\section{Full nonlinear theory}

\subsection{Quartet-metric gravity}

An underlying theory of gravity and space-time, whichever it might be, should inevitably manifest itself on an observable level as an effective field theory to match with GR and the conventional field theory for the ordinary matter. Thus in searching for such a fundamental theory, one should, conceivably, look first for the respective effective theory. The latter is to be generically characterized by a set of fields and symmetries ruling the interactions of the fields. Let us thus assume that the effective field theory of the extended gravity superseding GR is described by the dynamical fields of metric $g_{\mu \nu}$ and a scalar quartet $Q^{a}, a=0, \ldots, 3$, with an action

$S=\int L_{G}\left(g_{\mu \nu}, Q^{a}\right) \sqrt{-g} d^{4} x$,

where $g=\operatorname{det}\left(g_{\mu \nu}\right)$ and $L_{G}$ is an effective Lagrangian. ${ }^{4}$ The latter is assumed to have a $\mathrm{GC}$ form and to be invariant under a global internal Lorentz symmetry $S O(1,3)$ acting on the indices $a, b$, etc., with the invariant Minkowski symbol $\eta_{a b}$. It is understood that the signature of $\eta_{a b}$ determines the signature of $g_{\mu \nu}$. The physical meaning of the extra variables $Q^{a}$ will be clarified later on. Here we only mention that precisely these variables are responsible for the unified gravity $\mathrm{DM}$ and DE. Without loss of generality, $L_{G}$ may generically be decomposed into three parts depending on the appearance of the derivatives of metric:

$L_{G}=L_{g}\left(\partial_{\lambda} g_{\mu \nu}\right)+\Delta K\left(\partial_{\lambda} g_{\mu \nu}, Q^{a}\right)-V\left(Q^{a}\right)$.

An additional dependence directly on $g_{\mu \nu}$ to ensure GC is tacitly allowed, too. The part $L_{g}$ is a Lagrangian of the pure metric gravity, with $\Delta K$ and $V$ meaning, respectively, the hard/kinetic and soft/potential admixtures to the pure metric gravity. The order of the derivatives of $Q^{a}$ depends on the context (see below). At that, the appearance of $Q^{a}$ without derivatives is forbidden due to the assumed shift symmetry $Q^{a} \rightarrow Q^{a}+C^{a}$, with the arbitrary constants $C^{a}$. The

\footnotetext{
$\overline{4}$ Accordingly, in $d$ space-time dimensions there should occur $d$-pletmetric gravity.
} 
more tightly localized kinetic contributions are to be associated with the gravity DM, while the more loosely distributed potential ones with the gravity DE, roughly in compliance with their observational abilities to the spatial clusterization. The gravity DE and DM may thus be treated as the two facets of a single dark substance (DS), with the phenomenon of the unified origin of such a DS due to the common $Q^{a}$ referred to as the dark unification. Besides, after choosing a classical background and expanding the fields around the background, the scalar-quartet admixtures result in the appearance of the extra gravity degrees of freedom (d.o.f.'s) presenting the DS particles. We consider the various contributions to $L_{G}$ in more detail.

\subsection{Pure metric gravity}

Similarly to the plain GR, one may take for the pure metric gravity the minimal GC Lagrangian of the second order in the derivatives of metric:

$L_{g}=-\frac{\kappa_{g}^{2}}{2} R$.

Here $R$ is the Ricci curvature scalar, $\kappa_{g}=1 /\left(8 \pi G_{N}\right)^{1 / 2}$ the reduced Planck mass and $G_{N}$ the Newton constant. In what follows, we put $\kappa_{g}=1$. A GR modification, with a GC $L_{g}$ dependent only on metric, e.g., $f(R)$, is a priori conceivable, too. The more crucial GR extensions are due to the admixtures of $Q^{a}$ considered below.

\subsection{Hard/kinetic extension}

This kind of the GR extension is produced by the effective operators of the second order in the metric derivatives, likewise $L_{g}$. Instead of the derivatives of metric, one may equivalently use the Christoffel connection built of $g_{\mu \nu}$ :

$\Gamma_{\mu \nu}^{\lambda}=\frac{1}{2} g^{\lambda \kappa}\left(\partial_{\mu} g_{\nu \kappa}+\partial_{\nu} g_{\mu \kappa}-\partial_{\kappa} g_{\mu \nu}\right)$,

so that $\partial_{\nu} g_{\mu \rho}=g_{\rho \lambda} \Gamma_{\mu \nu}^{\lambda}+g_{\mu \lambda} \Gamma_{\rho \nu}^{\lambda}$. To preserve GC, the kinetic effective Lagrangian $\Delta K$ should depend on the difference of $\Gamma_{\mu \nu}^{\lambda}$ and an auxiliary affine connection $\gamma_{\mu \nu}^{\lambda}$ (for simplicity, symmetric) [12]:

$B_{\mu \nu}^{\lambda}=\Gamma_{\mu \nu}^{\lambda}-\gamma_{\mu \nu}^{\lambda}$

Such an auxiliary connection may minimally be taken as follows:

$\gamma_{\mu \nu}^{\lambda}=\left.\frac{\partial^{2} Q^{a}}{\partial x^{\mu} \partial x^{\nu}} \frac{\partial x^{\lambda}}{\partial Q^{a}}\right|_{Q^{a}=Q^{a}(x)} \equiv Q_{a}^{\lambda} \partial_{\mu} Q_{\nu}^{a}$,

where $Q_{\mu}^{a} \equiv \partial Q^{a} / \partial x^{\mu}$ and $Q_{a}^{\mu} \equiv \partial x^{\mu} /\left.\partial Q^{a}\right|_{Q^{a}=Q^{a}(x)}$, with $Q \equiv \operatorname{det}\left(\partial Q^{a} / \partial x^{\mu}\right) \neq 0$ or $\infty$ for the invertibility, $x^{\mu}=x^{\mu}\left(Q^{a}\right)$. It is the latter requirement which picks out $Q^{a}$ from other conceivable scalar fields. Assign to the extra variables $Q^{a}$ the dimension of length and the meaning as follows. Namely, assume that the vacuum is a kind of a physical medium modeled (ideally) by an affine-connected (i.e., possessing by an affine connection) manifold endowed with the absolute/affine (i.e., defined modulo the Lorentz transformations and translations) coordinates $Q^{a}$. At that, the conventional GR deals only with the relative/observer's coordinates $x^{\mu}$ undergoing the arbitrary (continuous) transformations among themselves. ${ }^{5}$ The quantities $q^{\alpha} \equiv \delta_{a}^{\alpha} Q^{a}$, insuring $\gamma_{\alpha \beta}^{\gamma}(q) \equiv 0$, define the distinguished observer's coordinates coinciding with $Q^{a}$. With respect to the arbitrary $x^{\mu}$, the distinguished $q^{\alpha}$ are the dynamical quantities, with $Q_{\mu}^{\alpha}=\partial q^{\alpha} / \partial x^{\mu}$ and $Q_{\alpha}^{\mu}=\partial x^{\mu} /\left.\partial q^{\alpha}\right|_{q^{\alpha}=q^{\alpha}(x)}$ being the frames relating these coordinates. In reality, the vacuum may have singularities, with the transition between the two kinds of coordinates being singular on a set of points (including, generally, the infinity). The extension of the set of the dynamical variables beyond the metric is tamed eventually by the expansion of the general gauge invariance/relativity onto the whole this set, leaving the total number of the independent field variables still equal to ten (as in the metric alone).

In the presence of $Q^{a}$, one may substitute a non-metrical affine connection $\gamma_{\mu \nu}^{\lambda}$ by a Christoffel one, $\gamma_{\mu \nu}^{\lambda}=\Gamma_{\mu \nu}^{\lambda}\left(\gamma_{\kappa \rho}\right)$, similarly to (4). It corresponds to an auxiliary metric (the affine metric):

$\gamma_{\mu \nu}=Q_{\mu}^{a} Q_{\nu}^{b} \eta_{a b}$

with the inverse $\gamma^{\mu \nu}=Q_{a}^{\mu} Q_{b}^{v} \eta^{a b}$, and $\gamma \equiv \operatorname{det}\left(\gamma_{\mu \nu}\right)=$ $-Q^{2} \neq 0$ or $\infty$ for the invertibility. By this token, formally $Q_{\mu}^{a}=\gamma_{\mu \lambda} \eta^{a b} Q_{b}^{\lambda}$ and $\gamma_{\mu \nu}=\gamma_{\mu \kappa} \gamma_{\mu \lambda} \gamma^{\kappa \lambda}$. In the distinguished coordinates $q^{\alpha}$, the affine metric coincides with the Minkowski symbol, $\gamma_{\alpha \beta}=\eta_{\alpha \beta}$ (simultaneously with $\left.\gamma_{\alpha \beta}^{\gamma}=0\right){ }^{6}$

Being given by the difference between the similarly transformed quantities, the field $B_{\mu \nu}^{\lambda}$ is a true tensor and, as such, may serve to construct a GC scalar Lagrangian. Introducing a complete set of the independent partial kinetic operators, bilinear in $B_{\mu \nu}^{\lambda}$ :

$$
\begin{array}{ll}
K_{1}=g^{\mu \nu} B_{\mu \kappa}^{\kappa} B_{\nu \lambda}^{\lambda}, & K_{2}=g_{\mu \nu} g^{\kappa \lambda} g^{\rho \sigma} B_{\kappa \lambda}^{\mu} B_{\rho \sigma}^{v}, \\
K_{3}=g^{\mu \nu} B_{\mu \nu}^{\kappa} B_{\kappa \lambda}^{\lambda}, & K_{4}=g_{\mu \nu} g^{\kappa \lambda} g^{\rho \sigma} B_{\kappa \rho}^{\mu} B_{\lambda \sigma}^{v}, \\
K_{5}=g^{\mu \nu} B_{\mu \kappa}^{\lambda} B_{\nu \lambda}^{\kappa}, &
\end{array}
$$

\footnotetext{
5 In a sense, it is proposed here a merging, at a next level, of the Newtonian and GR approaches to gravity and space-time.

${ }^{6}$ Stress that QMG is basically a one-metric theory. Using the affine metric $\gamma_{\mu \nu}$, instead of $Q^{a}$, is not obligatory, though technically convenient and geometrically clarifying.
} 
decompose $\Delta K$ as

$\Delta K=\frac{1}{2} \sum_{p=1}^{5} \varepsilon_{p} K_{p}$,

with some free parameters $\varepsilon_{p}, p=1, \ldots, 5$, presumably small, $\left|\varepsilon_{p}\right| \ll 1$. The two more second-derivative terms linear in $B_{\mu \nu}^{\lambda}, g^{\mu \nu} \nabla_{\lambda} B_{\mu \nu}^{\lambda}$ and $g^{\mu \nu} \nabla_{\mu} B_{\nu \lambda}^{\lambda}$, with $\nabla_{\lambda}$ a covariant derivative, are omitted due to the imposed invariance under the reflection $B_{\mu \nu}^{\lambda} \rightarrow-B_{\mu \nu}^{\lambda}$. This is the fully dynamical GR development of a semi-dynamical approach, the latter based on the explicit GR violation and a nondynamical $\hat{\gamma}_{\mu \nu}^{\lambda}$ [12].

\subsection{Soft/potential extension}

Such a GR extension, implementing the BEH mechanism for gravity, is produced by the effective operators, potentials, containing metric only without derivatives. To this end, take as the scalar fields the (dimensionless) $S O(1,3)$ decouplet [16]

$\Sigma^{a b}=g^{\mu \nu} Q_{\mu}^{a} Q_{v}^{b}$

incorporating the singlet

$\Sigma=\eta_{a b} \Sigma^{a b}=\gamma_{\mu \nu} g^{\mu \nu}$.

Substitute them equivalently by

$$
\begin{aligned}
\tilde{\Sigma}^{a b} & \equiv \Sigma^{a b}-\frac{1}{4} \eta^{a b} \Sigma, \\
\Sigma_{0} & \equiv \Sigma-4,
\end{aligned}
$$

so that $\tilde{\Sigma} \equiv \eta_{a b} \tilde{\Sigma}^{a b}=0$. We supplement $\Sigma_{0}$ by one more (dimensionless) scalar field

$\sigma=-\frac{1}{2} \ln \left|\operatorname{det}\left(\Sigma^{a b}\right)\right|=\ln \sqrt{-g} /|Q|=\ln \sqrt{-g} / \sqrt{-\gamma}$.

The latter field is precisely the one used previously for the scalar graviton/systolon [7-10]. The fields $\tilde{\Sigma}^{a b}, \Sigma_{0}$ and $\sigma$ prove to be homogeneous linear in the weak metric field in LA (see below) and are thus suitable for building a perturbative potential without a cosmological constant. The latter may be added, if desired, explicitly. Without loss of generality, the potential may be presented as follows:

$$
\begin{aligned}
V= & \frac{1}{8} m_{t}^{2}\left(\tilde{\Sigma}^{a b} \tilde{\Sigma}_{a b}-\frac{3}{4} \Sigma_{0}^{2}\right)+\frac{1}{8} m_{0}^{2} \Sigma_{0}^{2} \\
& \pm \frac{1}{4} m_{x}^{2} \Sigma_{0} \sigma+\frac{1}{2} m_{\sigma}^{2} \sigma^{2}+\Delta V\left(\tilde{\Sigma}^{a b}, \Sigma_{0}, \sigma\right),
\end{aligned}
$$

with $m_{t}, m_{0}, m_{x}$ and $m_{\sigma}$ some mass parameters, and $\Delta V$ a rest of the potential comprising the higher degrees of $\tilde{\Sigma}^{a b}$, $\Sigma_{0}$ and $\sigma$. The particular form of the first term of $V$ and the meaning of the parameters are to be justified by the compliance with the Fierz-Pauli LA Lagrangian (see below). The putative terms dependent only on $\gamma_{\mu \nu}$, such as $R\left(\gamma_{\mu \nu}\right)$, are absent due to the affine flatness of the space-time manifold, with $\gamma_{\mu \nu}$ reducing in the distinguished coordinates $q^{\alpha}$ to the Minkowskian $\eta_{\alpha \beta}$, hence $R\left(\gamma_{\mu \nu}\right)=0$.

\subsection{General diffeomorphism invariance}

Ultimately, the independent variables of the theory are $g_{\mu \nu}$ and $Q^{a}$. The Lie derivatives, defining the dynamical/active field transformations corresponding to the change of coordinates $\mathcal{D}_{\xi} x^{\lambda}=-\xi^{\lambda}$, with $\xi^{\lambda}$ an arbitrary shift vector, are as follows:

$$
\begin{aligned}
& \mathcal{D}_{\xi} Q^{a}=\xi^{\lambda} \partial_{\lambda} Q^{a} \equiv \xi^{\lambda} Q_{\lambda}^{a}, \\
& \mathcal{D}_{\xi} Q_{\mu}^{a}=\partial_{\mu} \mathcal{D}_{\xi} Q^{a}=Q_{\lambda}^{a} \partial_{\mu} \xi^{\lambda}+\xi^{\lambda} \partial_{\lambda} Q_{\mu}^{a}, \\
& \mathcal{D}_{\xi} Q_{a}^{\mu}=-Q_{a}^{\lambda} \partial_{\lambda} \xi^{\mu}+\xi^{\lambda} \partial_{\lambda} Q_{a}^{\mu}, \\
& \mathcal{D}_{\xi} g_{\mu \nu}=g_{\mu \lambda} \partial_{\nu} \xi^{\lambda}+g_{\nu \lambda} \partial_{\mu} \xi^{\lambda}+\xi^{\lambda} \partial_{\lambda} g_{\mu \nu}, \\
& \mathcal{D}_{\xi} g^{\mu \nu}=-g^{\mu \lambda} \partial_{\lambda} \xi^{\nu}-g^{\nu \lambda} \partial_{\lambda} \xi^{\mu}+\xi^{\lambda} \partial_{\lambda} g^{\mu \nu}
\end{aligned}
$$

(and similarly for $\gamma_{\mu \nu}$ and $\gamma^{\mu \nu}$ ). Henceforth, one can get the Lie derivatives of other quantities, e.g.,

$\mathcal{D}_{\xi} \sqrt{-g}=\partial_{\lambda}\left(\sqrt{-g} \xi^{\lambda}\right)$

(and similarly for $\sqrt{-\gamma}$ ), with $\sigma$ transforming thus as a scalar (as it should):

$\mathcal{D}_{\xi} \sigma=\xi^{\lambda} \partial_{\lambda} \sigma$

The same concerns the scalars $\Sigma^{a b}$ and $\Sigma$. The Lie derivative of any quantity may explicitly be expressed in a tensor form through replacing $\partial_{\mu}$ by a covariant derivative $\nabla_{\mu}$. In particular, one gets $\mathcal{D}_{\xi} g_{\mu \nu}=\nabla_{\mu} \xi_{v}+\nabla_{\nu} \xi_{\mu}$, where $\nabla_{\lambda} g_{\mu \nu}=0$ and $\xi_{\mu}=g_{\mu \lambda} \xi^{\lambda}$, etc. Due to GC, the full nonlinear theory, as containing only the dynamical fields, is automatically gauge invariant under the general diffeomorphisms (GDiff's). ${ }^{7}$ The latter ones reduce the number of the independent field components in $L_{G}$ up to ten (vs. six in GR). To account for the gauge degeneracy, a gauge fixing Lagrangian, $L_{F}$, appropriate for the problem at hand, is to be added. In particular, one may impose the same gauge conditions on the metric alone as in GR.

\subsection{Extended classical field equations}

Supplementing the gravity Lagrangian $L_{G}$ by the ordinary matter one, $L_{m}$, and varying the total action with respect to

\footnotetext{
7 This is what distinguishes the fully dynamical theory from a semidynamical model, where a residual gauge invariance/relativity is determined, under GC, through fixing the nondynamical fields [10].
} 
$\delta g^{\kappa \lambda}$ and $\delta Q^{a}\left(\delta Q_{\mu}^{a}=\nabla_{\mu} \delta Q^{a}\right)$, so that, in particular,

$$
\begin{aligned}
\delta \Sigma^{a b} & =Q_{\kappa}^{a} Q_{\lambda}^{b} \delta g^{\kappa \lambda}+2 g^{\kappa \lambda} Q_{\kappa}^{a} \delta Q_{\lambda}^{b}, \\
\delta \Sigma & =\eta_{a b} \delta \Sigma^{a b}=\gamma_{\kappa \lambda} \delta g^{\kappa \lambda}+g^{\kappa \lambda} \delta \gamma_{\kappa \lambda}, \\
\delta \gamma_{\kappa \lambda} & =\eta_{a b}\left(Q_{\kappa}^{a} \delta Q_{\lambda}^{b}+Q_{\lambda}^{a} \delta Q_{\kappa}^{b}\right), \\
\delta \sqrt{-\gamma} & =(1 / 2) \sqrt{-\gamma} \gamma^{\kappa \lambda} \delta \gamma_{\kappa \lambda} \\
\delta \sqrt{-g} & =-(1 / 2) \sqrt{-g} g_{\kappa \lambda} \delta g^{\kappa \lambda}, \\
\delta \sigma & =\delta \sqrt{-g} / \sqrt{-g}-\delta \sqrt{-\gamma} / \sqrt{-\gamma},
\end{aligned}
$$

with $\delta g_{\mu \nu}=-g_{\mu \kappa} g_{\nu \lambda} \delta g^{\kappa \lambda}$ (and similarly for $\gamma_{\mu \nu}$ ), one gets a pair of the coupled classical FE's for QMG in a generic form as follows:

$$
\begin{aligned}
G_{\mu \nu} \equiv R_{\mu \nu}-\frac{1}{2} & R g_{\mu \nu}=T_{\mu \nu}^{K}+T_{\mu \nu}^{V}+T_{\mu \nu}^{m} \\
\equiv T_{\mu \nu}^{D}+T_{\mu \nu}^{m} & \\
\nabla_{\kappa}\left(\Delta K^{\kappa \lambda} Q_{\lambda}^{a}\right)= & \nabla_{\kappa}\left(\left(\frac{\partial V}{\partial \Sigma^{c b}} g^{\kappa \lambda} \eta^{c a}\right.\right. \\
& \left.\left.\quad\left(\frac{1}{2} \frac{\partial V}{\partial \sigma} \gamma^{\kappa \lambda}+\frac{\partial L_{m}}{\partial \gamma_{\kappa \lambda}}\right) \delta_{b}^{a}\right) Q_{\lambda}^{b}\right),
\end{aligned}
$$

where $\Delta K^{\kappa \lambda} \equiv \delta \Delta K / \delta \gamma_{\kappa}$, with $\delta / \delta \gamma_{\kappa \lambda}$ a total variational derivative with respect to $\gamma_{\kappa \lambda}$. The variables $\tilde{\Sigma}^{a b}$ and $\Sigma_{0}$ are assumed to be expressed through $\Sigma^{a b}$. The 1.h.s.'s of FE's depend on the second derivatives of metric, while the r.h.s.'s only on the first derivatives. In the spirit of DM, $L_{m}$ is assumed to depend on $Q^{a}$ (if any) exclusively through $\gamma_{\kappa} \lambda$, without its derivatives. To eliminate the gauge ambiguity, in solving FE's one should first fix the coordinates by imposing an appropriate gauge condition, which will tacitly be understood. In reality, fixing the coordinate to maximally simplify the metric FE's proves to be the most appropriate. ${ }^{8}$ The 1.h.s. in the upper line of (19) is the gravity tensor $G_{\mu \nu}$ due to $L_{g}$, with $T_{\mu \nu}^{D} \equiv \Delta T_{\mu \nu}^{K}+T_{\mu \nu}^{V}$ in the r.h.s. treated as the energy-momentum tensor of DS. This is, in essence, the raison d'être for associating the admixtures due to $Q^{a}$ with DS. The kinetic contribution to $T_{\mu \nu}^{D}$ is

$\Delta T_{\mu \nu}^{K} \equiv \frac{1}{2} \sum_{p=1}^{5} \varepsilon_{p} T_{p \mu \nu}^{K}$

where $T_{p \mu \nu}^{K}$ are the partial contributions due to $K_{p}$ :

$T_{p \mu \nu}^{K}=\frac{2}{\sqrt{-g}} \frac{\delta\left(\sqrt{-g} K_{p}\right)}{\delta g^{\mu \nu}}$,

with $\delta / \delta g^{\mu \nu}$ designating a total variational derivative with respect to $g^{\mu \nu}$. A similar expression holds for the canonical

\footnotetext{
${ }^{8}$ On the contrary, choosing the distinguished coordinates $q^{\alpha}$, superficially convenient, one would lose such a freedom of simplifying the metric FE's, what proves to be crucial in looking for the explicit solutions.
}

energy-momentum tensor $T_{\mu \nu}^{m}$ of the ordinary matter. Likewise, the potential contribution to $T_{\mu \nu}^{D}$ is

$T_{\mu \nu}^{V}=-2 \frac{\partial V}{\partial \Sigma^{a b}} Q_{\mu}^{a} Q_{\nu}^{b}+\left(\frac{\partial V}{\partial \sigma}+V\right) g_{\mu \nu}$.

In particular, a nonzero constant part of $V$ would correspond to a cosmological term. Due to the reduced Bianchi identity, $\nabla_{\mu} G^{\mu \nu}=0$, the total energy-momentum tensor, $T_{\mu \nu}$, should be covariantly conserved:

$\nabla_{\mu} T^{\mu \nu} \equiv \nabla_{\mu}\left(T_{m}^{\mu \nu}+T_{D}^{\mu \nu}\right)=0$.

Assuming $L_{m}$ to be independent of $Q^{a}$, one conventionally gets with account for the ordinary matter FE's that $\nabla_{\mu} T_{m}^{\mu \nu}=$ 0 . In this case (or in the case of matter vacuum), the DS contribution should separately be covariantly conserved.

The system of FE's (19) presents the essence of the fully dynamical theory. Namely, it may be said that (19) determines in a self-consistent manner a two-level structure of the space-time manifold: a basic affine structure and a fine metric one. The first line of FE's (19), obtained by varying only $g_{\mu \nu}$, remains the same independently of whether $Q^{a}$ are dynamical or not. Thus the first equation (19), determining a metric structure at a given affine one, is unchanged compared to the semi-dynamical model. The second equation acts vice versa, determining the back reaction of the metric structure on the affine one. At the very least, a self-consistent solution may be looked for by means of the consecutive approximations starting from a putative solution (under convergence of the procedure). The account for the full dynamics should restrict a prior freedom of choosing an otherwise arbitrary nondynamical background in the semi-dynamical approach. For a special case, this is worked out below.

\subsection{Minimal extension}

As a minimal kinetic contribution to the quartet GR extension, one may consider the operator

$K_{1}=g^{\mu \nu} \partial_{\mu} \ln \frac{\sqrt{-g}}{|Q|} \partial_{\nu} \ln \frac{\sqrt{-g}}{|Q|}$

where $|Q| \equiv\left|\operatorname{det}\left(Q_{\mu}^{a}\right)\right|=\sqrt{-\gamma}$. This restricted case presents a fully dynamical generalization of the semidynamical model for the scalar-graviton/systolon DM, with a nondynamical $\hat{\gamma}$ and the explicit GR violation [7-10]. Under the GR extension exclusively through $K_{1}(\sigma)$ and $V(\sigma)$, there is a parity between the numbers of the independent variables in the full nonlinear theory and in its LA, both being seven (see below). The quartet enters only through $\sqrt{-\gamma}=|Q|$, irrespective of $g_{\mu \nu}$. Under the metric variation, $\gamma$ thus remains unvaried. Hence the scalar-graviton part of the metric FE's in vacuum, obtained previously in the 
semi-dynamical model $[9,10]$,

$\varepsilon_{1} \nabla^{\kappa} \nabla_{\kappa} \sigma+\partial V / \partial \sigma=\Lambda_{0} e^{-\sigma}$,

remains unchanged. In the above, $\Lambda_{0}$ is an integration constant arising due to the reduced Bianchi identity. To restrict himself by a conventionally adopted scalar field $\sigma$ decaying at the spatial infinity, one should put $\Lambda_{0}=0$. On the other hand, with $K_{1}$ dependent on $Q^{a}$ exclusively through $\sigma$, the quartet part of FE's in vacuum becomes as follows:

$\nabla_{\lambda}\left(\left(\varepsilon_{1} \nabla^{\kappa} \nabla_{\kappa} \sigma+\partial V / \partial \sigma\right) Q_{a}^{\lambda}\right)=0$.

Combining the two equations one gets the consistency condition:

$\Lambda_{0} \nabla_{\lambda}\left(e^{-\sigma} Q_{a}^{\lambda}\right)=\left(\Lambda_{0} / \sqrt{-g}\right) \partial_{\lambda}\left(\sqrt{-g} e^{-\sigma} Q_{a}^{\lambda}\right)=0$.

Several static spherically symmetric solutions of the metric part of FE's at $V=0$ in the semi-dynamical model are presented in [10]. Their prolongation to the fully dynamical theory is as follows.

\subsubsection{Compact cosmic objects}

Let first $\Lambda_{0}=0$. In this case, $Q^{a}$ remains still unrestricted except for a singular in the spatial origin $\left|\operatorname{det}\left(Q_{\mu}^{a}\right)\right|=\sqrt{-\gamma}$. The latter is given through metric and $\sigma$ by an exact solution to the first part of FE's (19) under the regular at the spatial infinity boundary conditions, similarly to the semidynamical model. The proper affine structure can be chosen to be $\delta$-wise flat (i.e., flat with exclusion of the time-like line spreading through the spatial origin). ${ }^{9}$ Hence, a wide class of the compact cosmic objects, filled with the scalar gravitons/systolons as DM, found in the semi-dynamical model remains still appropriate in the fully dynamical theory. In distinction with the BH's of GR, these objects are allowed even in the absence of the ordinary matter, being caused by singularities of the space-time itself (henceforth we call this a dark fracture (DF) [10]). Modifying BH's of GR, DF's may possess by a quite different structure of the event horizon. Note that DF's can still be mimicked by BH's of GR with a (massless) scalar field (though of unknown nature). One more kind of the cosmic objects in vacuum peculiar, as DF's, exclusively to QMG (and to its semi-dynamical counterpart) is as follows.

\subsubsection{Extended cosmic objects}

Let now $\Lambda_{0} \neq 0$. This case corresponds to the extended cosmic objects in the vacuum, the dark halos (DH's), peculiar exclusively to QMG (or to its semi-dynamical counterpart). Under a regular boundary condition in the spatial origin, $\gamma$ is

\footnotetext{
9 Conceivably, this ambiguity may be eliminated by treating DF's as a limiting case of the more general cosmic objects (see below).
}

determined in the chosen (due to fixing a gauge for metric) observer's coordinates $x^{\mu}$ from the first part of FE's (19) by the metric and $\sigma$, as in the semi-dynamical model $[9,10]$. In view of (13), $e^{-\sigma}=\sqrt{-\gamma} / \sqrt{-g}$, Eq. (27) gives

$\partial_{\lambda}\left(\sqrt{-\gamma} Q_{a}^{\lambda}\right)=0$

with the metric $g_{\mu \nu}$ falling off. We consider the static spherically symmetric solutions to the metric FE's, with $\gamma=\gamma(r)$, $r$ the distance from the origin. Transforming the expressions from the original coordinates $x^{\mu}=\left(x^{0}, x^{m}\right), m=1,2,3$, to the distinguished ones $q^{\alpha}=\left(q^{0}, q^{m}\right)$, given by $q^{0}=$ $\sqrt{-\gamma} x^{0}, q^{m}=x^{m}$, wherein $\gamma=-1$, one gets the solution $Q^{a}=\delta_{\alpha}^{a} q^{\alpha}$, implying in the previously fixed coordinates $x^{\mu}$ the scalar quartet $Q^{a}=\left(Q^{(0)}, Q^{A}\right)$ :

$Q^{(0)}=\sqrt{-\gamma} x^{0}, \quad Q^{A}=\delta_{k}^{A} x^{k}$,

where $A=1,2,3$. For $\partial_{\mu} Q^{a} \equiv Q_{\mu}^{a}=\left(Q_{\mu}^{(0)}, Q_{\mu}^{A}\right)$ one thus gets

$Q_{0}^{(0)}=\sqrt{-\gamma}, \quad Q_{m}^{(0)}=\frac{-\gamma^{\prime}}{2 \sqrt{-\gamma}} x^{0} n_{m}$,

$Q_{0}^{A}=0, \quad Q_{m}^{A}=\delta_{m}^{A}$,

and then for its inverse $Q_{a}^{\mu}=\left(Q_{(0)}^{\mu}, Q_{A}^{\mu}\right)$ :

$$
\begin{aligned}
Q_{(0)}^{0} & =\frac{1}{\sqrt{-\gamma}}, \quad Q_{(0)}^{m}=0, \\
Q_{A}^{0} & =\frac{-\gamma^{\prime}}{2 \gamma} x^{0} \delta_{A}^{k} n_{k}, \quad Q_{A}^{m}=\delta_{A}^{m},
\end{aligned}
$$

where $\gamma^{\prime}=d \gamma / d r, n^{m}=x^{m} / r$ and $n_{m}=\delta_{m k} n^{k}$. Equation (28) is satisfied, indeed. The affine metric $\gamma_{\mu \nu}=$ $Q_{\mu}^{a} Q_{v}^{b} \eta_{a b}$ in the original coordinates is as follows:

$\gamma_{00}=-\gamma, \quad \gamma_{m 0}=\gamma_{0 m}=-\frac{1}{2} \gamma^{\prime} x^{0} n_{m}$,

$\gamma_{m l}=-\delta_{m l}-\frac{1}{4 \gamma} \gamma^{\prime 2}\left(x^{0}\right)^{2} n_{m} n_{l}$

with $\operatorname{det}\left(\gamma_{\mu \nu}\right)=\gamma(r)$, indeed. In line with $\gamma$, this metric is irregular at the spatial infinity. In the arbitrary $x^{\mu}$, all this fields correspond to $Q^{a}$ obtained according to the transformation law for scalars. Clearly, in the distinguished coordinates $q^{\alpha}$, where $\gamma=-1$, the affine metric reduces to $\gamma_{\alpha \beta}=\eta_{\alpha \beta}$, the Minkowski symbol, with $\gamma_{\alpha \beta}^{\gamma}=0$. The DH space-time possesses thus by the flat affine structure as the pseudo-Euclidean space-time, but, in distinction with the latter, by a non-flat metric structure. The principal difference between the two space-times is due to a singularity of DH's at the spatial infinity. The metric structure, obtained in the semi-dynamical model, remains still valid (supplemented by the proper affine structure) in the fully dynamical theory. Hence, all the properties of the galaxy soft-core DH's, built exclusively of the scalar gravitons/systolons as DM, arrived at previously, remain in force. With account 
for the asymptotic $1 / r$-behavior of the attractive force in DH's $[9,10]$, the latter ones constitute the separate asymptotically confined "mini-universes" (in neglect by the edge effects).

\subsubsection{Compact-extended cosmic objects}

At $\Lambda_{0} \neq 0$, there exist the (approximate) vacuum solutions to the extended FE's, singular in the spatial origin and at the spatial infinity, and possessing, conceivably, by a $\delta$-wise flat affine structure. These solutions present very peculiar cosmic objects, the dark lacunas (DL's) [10], with the compact cores and extended tails, interpolating between DF's and DH's. Having DF's in their origin, DL's could model the galaxies (poor of the ordinary matter). Studying such the cosmic objects (and their matter, rotation and other modifications) to model the real galaxies could present a future challenge for QMG.

\section{Weak-field limit}

\subsection{Weak-field expansion}

The physics content of a nonlinear field theory, as the quantum one, is reflected by its WF limit. To this end, considering the expansion around some backgrounds $\bar{g}_{\mu \nu}$ and $\hat{Q}^{a}$, with $g_{\mu \nu} \equiv \bar{g}_{\mu \nu}+h_{\mu \nu}$ and $Q^{a} \equiv \hat{Q}^{a}+\chi^{a}$, one gets up to the first order in the deviations

$$
\begin{aligned}
g^{\mu \nu} & \equiv \bar{g}^{\mu \nu}-h^{\mu \nu}=\bar{g}^{\mu \nu}-\bar{g}^{\mu \kappa} \bar{g}^{\nu \lambda} h_{\kappa \lambda}, \\
Q_{\mu}^{a} & =\hat{Q}_{\mu}^{a}+\partial_{\mu} \chi^{a}, \quad Q_{a}^{\mu}=\hat{Q}_{a}^{\mu}-\eta_{a b} \hat{\gamma}^{\mu \lambda} \partial_{\lambda} \chi^{b},
\end{aligned}
$$

with $\hat{Q}_{\mu}^{a}=\partial_{\mu} \hat{Q}^{a}, \hat{Q}_{a}^{\mu}$ the inverse to $\hat{Q}_{\mu}^{a}, \hat{\gamma}^{\mu \nu}=\hat{Q}_{a}^{\mu} \hat{Q}_{b}^{v} \eta^{a b}$, and $\left|h_{\mu \nu}\right|,\left|\chi^{a}\right| \ll 1$. By default, the indices in the WF limit are raised and lowered by $\bar{g}^{\mu \nu}$ and $\bar{g}_{\mu \nu}$, respectively, unless stated otherwise. The parts $\hat{Q}^{a}$ and $\chi^{a}$ may be associated with a mean value and the fluctuations of the absolute/affine coordinates $Q^{a}$ (or thus the distinguished $q^{\alpha}=\delta_{a}^{\alpha} Q^{a}$ ) relative to the smoothed observer's ones $x^{\mu}$. The latter coordinates are assumed to be fixed by a suitable gauge condition. Ultimately, $\hat{Q}^{a}$ and $\chi^{a}$ determine, respectively, the classical and quantum manifestations of DS. In terms of these, one has

$$
\Sigma^{a b}=\hat{Q}_{\kappa}^{a} \hat{Q}_{\lambda}^{b}\left(\bar{g}^{\kappa \lambda}-h^{\kappa \lambda}\right)+\bar{g}^{\kappa \lambda}\left(\hat{Q}_{\kappa}^{a} \partial_{\lambda} \chi^{b}+\hat{Q}_{\kappa}^{b} \partial_{\lambda} \chi^{a}\right) .
$$

To clarify the space-time structure of the WF theory in a GC form it is more appropriate to deal exclusively with the spacetime notation. To this end, introducing $\chi^{\mu} \equiv \hat{Q}_{a}^{\mu} \chi^{a}\left(\chi^{a}=\right.$ $\left.\hat{Q}_{\kappa}^{a} \chi^{\kappa}\right)$ one gets for $\Sigma^{\mu \nu} \equiv \hat{Q}_{a}^{\mu} \hat{Q}_{b}^{v} \Sigma^{a b}\left(\Sigma^{a b}=\hat{Q}_{\kappa}^{a} \hat{Q}_{\lambda}^{b} \Sigma^{\kappa \lambda}\right)$ :

$$
\Sigma^{\mu \nu}=\bar{g}^{\mu \nu}-h^{\mu \nu}+\bar{g}^{\mu \lambda} \hat{\nabla}_{\lambda} \chi^{\nu}+\bar{g}^{\nu \lambda} \hat{\nabla}_{\lambda} \chi^{\mu},
$$

where

$\hat{\nabla}_{\lambda} \chi^{\mu} \equiv\left(\delta_{\kappa}^{\mu} \partial_{\lambda}+\hat{Q}_{a}^{\mu} \partial_{\lambda} \hat{Q}_{\kappa}^{a}\right) \chi^{\kappa}$

is nothing but a covariant derivative with respect to $\hat{\gamma}_{\mu \nu}$, so that $\hat{\nabla}_{\lambda} \hat{\gamma}_{\mu \nu}=0$ (but not for $\bar{g}_{\mu \nu}$ ). Similarly, for

$\gamma_{\mu \nu}=\hat{Q}_{\mu}^{a} \hat{Q}_{\nu}^{b} \eta_{a b}+\left(\hat{Q}_{\mu}^{a} \partial_{\nu} \chi^{b}+\hat{Q}_{\nu}^{a} \partial_{\mu} \chi^{b}\right) \eta_{a b}$

one gets

$\gamma_{\mu \nu}=\hat{\gamma}_{\mu \nu}+\hat{\gamma}_{\mu \lambda} \hat{\nabla}_{\nu} \chi^{\lambda}+\hat{\gamma}_{\nu \lambda} \hat{\nabla}_{\mu} \chi^{\lambda}$

where $\hat{\gamma}_{\mu \nu}=\hat{Q}_{\mu}^{a} \hat{Q}_{v}^{b} \eta_{a b}$, with $\hat{\gamma}^{\mu \nu}=\hat{Q}_{a}^{\mu} \hat{Q}_{b}^{v} \eta^{a b}$ being its inverse.

\section{2 $\mathrm{BEH}$ mechanism for extended gravity}

Now, consider some arbitrary backgrounds $\bar{g}_{\mu \nu}$ and $\hat{Q}^{a}$ (or, equivalently, $\hat{\gamma}_{\mu \nu}$ ) with the coordinates fixed by a background gauge condition. Under the additional infinitesimal coordinate transformations through an arbitrary gauge parameter $\zeta^{\lambda}, \mathcal{D}_{\zeta} x^{\lambda}=-\zeta^{\lambda}$, leaving by construction the backgrounds invariant, one can find from (15) and (33) for $h_{\mu \nu}=\bar{g}_{\mu \kappa} \bar{g}_{\nu \lambda} h^{\kappa \lambda}$ and $\chi^{\mu}$ the ensuing gauge transformations

$\mathcal{D}_{\zeta} h_{\mu \nu}=\bar{\nabla}_{\mu} \zeta_{\nu}+\bar{\nabla}_{\nu} \zeta_{\mu}$

$\mathcal{D}_{\zeta} \chi^{\mu}=\zeta^{\mu}$.

Here $\zeta_{\mu}=\bar{g}_{\mu \lambda} \zeta^{\lambda}$ and $\bar{\nabla}_{\lambda}$ is a covariant derivative with respect to $\bar{g}_{\mu \nu}$, so that $\bar{\nabla}_{\lambda} \bar{g}_{\mu \nu}=0$ (but not for $\hat{\gamma}_{\mu \nu}$ ). Fixing further the particular gauge $\zeta^{\lambda}=-\chi^{\lambda}$ one arrives at the quartet field disappearance:

$\chi^{\mu} \rightarrow \chi^{\prime \mu}=0$

in favor of the metric field redefinition:

$$
\begin{aligned}
h_{\mu \nu} & \rightarrow h_{\mu \nu}^{\prime}=h_{\mu \nu}-\left(\bar{g}_{\mu \lambda} \bar{\nabla}_{\nu} \chi^{\lambda}+\bar{g}_{\nu \lambda} \hat{\nabla}_{\mu} \chi^{\lambda}\right), \\
h & \rightarrow h^{\prime} \equiv \bar{g}^{\mu \nu} h_{\mu \nu}^{\prime}=h-2 \bar{\nabla}_{\lambda} \chi^{\lambda} .
\end{aligned}
$$

It follows henceforth that after such transformations the whole Lagrangian $L_{G}$ (and, in particular, $L_{g}$ ) gets dependent only on $h_{\mu \nu}^{\prime}$. Due to $\gamma_{\mu \nu} \rightarrow \hat{\gamma}_{\mu \nu}$, the fully dynamical theory in the WF limit reduces thus to the semi-dynamical model, with the redefined metric field $h_{\mu \nu}^{\prime}$ and the given nondynamical affine connection $\hat{\gamma}_{\mu \nu}^{\lambda}\left(\hat{\gamma}_{\kappa \lambda}\right)$. In particular, presenting $\sigma$ as

$\sigma=-\frac{1}{2} \ln \left|\operatorname{det}\left(\Sigma^{\mu v}\right) \hat{Q}^{2}\right|$

one gets

$\sigma=\ln \sqrt{-\bar{g}} / \sqrt{-\hat{\gamma}}+\frac{1}{2} h^{\prime}$,

as in the minimal semi-dynamical model, with $\bar{g} \equiv \operatorname{det}\left(\bar{g}_{\mu \nu}\right)$ and $\hat{\gamma} \equiv \operatorname{det}\left(\hat{\gamma}_{\mu \nu}\right)=-\hat{Q}^{2}$. The similar consideration remains true with account for a matter Lagrangian, $L_{m}$. 
This presents an implementation of the BEH mechanism for the extended gravity: the gauge $\zeta^{\lambda}=-\chi^{\lambda}$ totally absorbs $\chi^{\lambda}$ in favor of the four additional gravity d.o.f.'s contained in $h_{\mu \nu}^{\prime}$, chosen as a new dynamical variable. Due to the gauge invariance, the WF theory in an arbitrary gauge, a WF remnant of $L_{F}$, still describes the same ten d.o.f.'s (the six tensor ones originating ultimately from the metric field $h_{\mu \nu}$ ) and the four scalar and vector ones (originating from the quartet $\chi^{\mu}$ ) incorporating, possibly, the ghosts. Further clarifying the nature of these d.o.f.'s (propagating or not, ghost or not) depends on a residual gauge invariance/relativity determined, in turn, by the Lagrangian parameters. This question is addressed below.

\subsection{Linearized approximation}

Consider now the simplest version of the WF theory, when the metric and quartet backgrounds are globally flat, presenting evidently a solution to the vacuum FE's. At least, this can be treated as an approximation, in neglect by the space-time curvature, in a region around a nonsingular space-time point. Moreover, choose the distinguished observer's coordinates for the background, $\hat{q}^{\alpha}=\delta_{a}^{\alpha} \hat{Q}^{a}\left(\hat{Q}^{a}=\delta_{\alpha}^{a} \hat{q}^{\alpha}\right)$, where there simultaneously is fulfilled

$\hat{Q}_{\alpha}^{a}=\partial_{\alpha} \hat{Q}^{a}=\delta_{\alpha}^{a}, \quad \bar{g}_{\alpha \beta}=\hat{\gamma}_{\alpha \beta}=\eta_{\alpha \beta}$,

with $\eta_{\alpha \beta}$ the Minkowski symbol, by means of which the indices are operated upon. In view of $\Sigma^{\alpha \beta}=\eta^{\alpha \beta}-h^{\prime \alpha \beta}$, where

$$
\begin{gathered}
h_{\alpha \beta}^{\prime}=h_{\alpha \beta}-\left(\partial_{\alpha} \chi_{\beta}+\partial_{\beta} \chi_{\alpha}\right), \\
h^{\prime} \equiv \eta^{\alpha \beta} h_{\alpha \beta}^{\prime}=h-2 \partial_{\gamma} \chi^{\gamma},
\end{gathered}
$$

it follows that

$$
\begin{gathered}
\tilde{\Sigma}^{\alpha \beta}=-\left(h^{\alpha \beta}-\frac{1}{4} \eta^{\alpha \beta} h^{\prime}\right), \\
\Sigma_{0}=-h^{\prime}, \quad \sigma=\frac{1}{2} h^{\prime} .
\end{gathered}
$$

Note that $h_{\alpha \beta}^{\prime}$ remains invariant under the gauge transformations (39), while $\chi^{\alpha}$ disappears at $\zeta^{\alpha}=-\chi^{\alpha}$. Due to $\bar{\Gamma}_{\alpha \beta}^{\gamma}=\hat{\gamma}_{\alpha \beta}^{\gamma}=0$, one also gets

$B_{\alpha \beta}^{\gamma}=\frac{1}{2} \eta^{\gamma \delta}\left(\partial_{\alpha} h_{\beta \delta}^{\prime}+\partial_{\beta} h_{\alpha \delta}^{\prime}-\partial_{\delta} h_{\alpha \beta}^{\prime}\right)$.

Under the requirement of the two background connections being zero, the (nonlinear) WF limit is nothing but LA.

We choose a complete (up to the total derivatives) set of the second-order partial kinetic operators in an obvious notation as follows:

$$
\begin{aligned}
& K_{t}=\left(\partial_{\gamma} h_{\alpha \beta}^{\prime}\right)^{2}, \quad K_{s}=\left(\partial_{\alpha} h^{\prime}\right)^{2}, \\
& K_{v}=\left(\partial^{\beta} h_{\alpha \beta}^{\prime}\right)^{2}, \quad K_{x}=\partial^{\alpha} h_{\alpha \beta}^{\prime} \partial^{\beta} h^{\prime} .
\end{aligned}
$$

Expanding the Lagrangian up to the second order in the redefined metric field and eliminating $K_{t}$ in favor of $L_{g}$ one gets

$$
\begin{aligned}
L_{G}= & \left(1+\varepsilon_{t}\right) L_{g}+\Delta K_{v s} \\
& -\frac{1}{8} m_{t}^{2}\left(\left(h_{\alpha \beta}^{\prime}\right)^{2}-h^{\prime 2}\right)-\frac{1}{8} m_{s}^{2} h^{\prime 2},
\end{aligned}
$$

where

$m_{s}^{2} \equiv m_{0}^{2} \mp m_{x}^{2}+m_{\sigma}^{2}$

and

$$
\begin{aligned}
L_{g} & =\frac{1}{8}\left(K_{t}-2 K_{v}+2 K_{x}-K_{s}\right), \\
\Delta K_{v s} & =\frac{1}{8}\left(\varepsilon_{v} K_{v}+\varepsilon_{x} K_{x}+\varepsilon_{s} K_{s}\right) .
\end{aligned}
$$

In what follows, we will intently preserve the primes to stress that the metric field at hand is the redefined one, absorbing $\chi^{\alpha}$

The five-to-four projection for the partial constants is as follows:

$$
\begin{aligned}
& \varepsilon_{t}=3 \varepsilon_{4}-\varepsilon_{5}, \quad \varepsilon_{v}=4\left(\varepsilon_{2}+\varepsilon_{4}\right) \\
& \varepsilon_{x}=-2\left(\varepsilon_{2}-\varepsilon_{3}+3 \varepsilon_{4}-\varepsilon_{5}\right) \\
& \varepsilon_{s}=\varepsilon_{1}+\varepsilon_{2}-\varepsilon_{3}+3 \varepsilon_{4}-\varepsilon_{5}
\end{aligned}
$$

Inverting, one gets

$$
\begin{aligned}
& \varepsilon_{1}=\varepsilon_{s}+\frac{1}{2} \varepsilon_{x}, \quad \varepsilon_{2}=\frac{1}{4} \varepsilon_{v}-\lambda, \\
& \varepsilon_{3}=\frac{1}{4} \varepsilon_{v}+\frac{1}{2} \varepsilon_{x}+\varepsilon_{t}-\lambda, \quad \varepsilon_{4}=\lambda, \\
& \varepsilon_{5}=-\varepsilon_{t}+3 \lambda,
\end{aligned}
$$

where $\lambda$ is a small arbitrary parameter. The latter may equivalently be redefined in favor of a linear combination of itself and $\varepsilon_{v}, \varepsilon_{x}$, and $\varepsilon_{t}$. The presence of such an undetermined parameter results in the partial decoupling of the full nonlinear theory and its WF limit, allowing one, in principle, to vary the former under choosing the latter. Clearly, the pair $\left(\varepsilon_{1}, \varepsilon_{S}\right)$ constitutes a closed uniquely invertible subset of all the parameters, with the pure-scalar operator $K_{1}$ being in this sense special. Its minimal modification without changing LA may be obtained by adding in the full nonlinear theory the terms proportional to $\lambda$.

Though after choosing the gauge $\zeta^{\alpha}=-\chi^{\alpha}$ the coordinates in LA are already fixed leaving, generally, the ten independent variables $h_{\mu \nu}^{\prime}, L_{G}$ may still possess in LA a residual gauge invariance further reducing the number of d.o.f.'s. Namely, under the Diff's

$\mathcal{D}_{\varphi} h_{\alpha \beta}^{\prime}=\partial_{\alpha} \varphi_{\beta}+\partial_{\beta} \varphi_{\alpha}, \quad \mathcal{D}_{\varphi} h^{\prime}=2 \partial_{\gamma} \varphi^{\gamma}$, 
one gets (up to total derivatives)

$$
\begin{aligned}
& \mathcal{D}_{\varphi} K_{t}=-2\left(\partial_{\alpha} \varphi_{\beta}+\partial_{\beta} \varphi_{\alpha}\right) \partial^{2} h^{\prime \alpha \beta}, \\
& \mathcal{D}_{\varphi} K_{v}=-\left(\partial_{\alpha} \varphi_{\beta}+\partial_{\beta} \varphi_{\alpha}\right) \partial^{2} h^{\prime \alpha \beta}-\partial_{\gamma} \varphi^{\gamma} \partial_{\alpha} \partial_{\beta} h^{\prime \alpha \beta}, \\
& \mathcal{D}_{\varphi} K_{x}=-\partial_{\gamma} \varphi^{\gamma}\left(\partial^{2} h^{\prime}+\partial_{\alpha} \partial_{\beta} h^{\prime \alpha \beta}\right), \\
& \mathcal{D}_{\varphi} K_{s}=-2 \partial_{\gamma} \varphi^{\gamma} \partial^{2} h^{\prime},
\end{aligned}
$$

where $\partial^{2} \equiv \eta^{\alpha \beta} \partial_{\alpha} \partial_{\beta}$, as well as

$$
\begin{aligned}
\mathcal{D}_{\varphi}\left(h_{\alpha \beta}^{\prime}\right)^{2} & =2\left(\partial_{\alpha} \varphi_{\beta}+\partial_{\beta} \varphi_{\alpha}\right) h^{\prime \alpha \beta}, \\
\mathcal{D}_{\varphi} h^{\prime 2} & =4 \partial_{\gamma} \varphi^{\gamma} h^{\prime} .
\end{aligned}
$$

It follows henceforth that $L_{g}$ is by the very construction GDiff-invariant, $\mathcal{D}_{\varphi} L_{g}=0$, whereas the rest of $L_{G}$ (49) is always GDiff-variant. Most generally thus, LA possesses no residual Diff's. Inclusion of the four scalar components in the original Lagrangian results in LA in the four more gravity d.o.f.'s. With no residual Diff's, all the ten d.o.f.'s are thus propagating. This may cause some theoretical problems related with the appearance of the ghost vector graviton, e.g., the classical instabilities [6]. To abandon this, one may impose ab initio some restrictions on the parameters of $L_{G}$, discussed below.

\subsection{Residual transverse-diffeomorphism invariance}

To consistently exclude the vector graviton let us require

$\varepsilon_{v}=4\left(\varepsilon_{2}+\varepsilon_{4}\right)=0, m_{t}=0$.

While the first requirement is necessary, the appearance of the second one is, in a sense, sufficient (see, later on). ${ }^{10}$ The Lagrangian in LA now becomes

$$
\begin{aligned}
L_{G} & =\left(1+\varepsilon_{t}\right) L_{g}+\Delta K_{s}-\frac{1}{8} m_{s}^{2} h^{\prime 2}, \\
\Delta K_{s} & =\frac{1}{8}\left(\tilde{\varepsilon}_{x} K_{x}+\tilde{\varepsilon}_{s} K_{s}\right),
\end{aligned}
$$

with the same $\varepsilon_{t}$, and the reduced partial constants as follows:

$\tilde{\varepsilon}_{x}=2\left(\varepsilon_{3}-2 \varepsilon_{4}+\varepsilon_{5}\right)$,

$\tilde{\varepsilon}_{s}=\varepsilon_{1}-\left(\varepsilon_{3}-2 \varepsilon_{4}+\varepsilon_{5}\right)$.

It follows from (55) that the residual gauge symmetry of $L_{G}$ in LA increases under such the constraints from the no-Diff case up to the three-parameter TDiff:

TDiff : $\partial_{\gamma} \varphi^{\gamma}=0$.

The two constraints (57) select the most general theory possessing in LA by no explicit problems. On the one hand, the constraints result in the appearance of TDiff. On the other hand, TDiff ensures these constraints to be natural in the 't Hooft's sense of increasing the residual symmetry. Due to

\footnotetext{
${ }^{10}$ Moreover, under $m_{s} \neq 0$, imposed from the DS considerations, the simultaneous fulfillment of $m_{t} \neq 0$ may result in ghosts [6].
}

the increased symmetry, such constraints may survive under the radiative corrections. ${ }^{11}$

Under TDiff, to remove in LA the arising gauge ambiguity one should impose on $h_{\alpha \beta}^{\prime}$ at the classical level an extra gauge condition, e.g., $[3,4]$ :

$\partial_{\alpha} \partial^{\gamma} h_{\beta \gamma}^{\prime}-\partial_{\beta} \partial^{\gamma} h_{\alpha \gamma}^{\prime}=0$.

Decomposing $h_{\alpha \beta}^{\prime}$ as

$h_{\alpha \beta}^{\prime}=\tilde{h}_{\alpha \beta}^{\prime}+\frac{1}{4} \eta_{\alpha \beta} h^{\prime}$,

with $\tilde{h}^{\prime} \equiv \eta^{\alpha \beta} \tilde{h}_{\alpha \beta}^{\prime}=0$, one sees that $h^{\prime}$ is unrestricted by the gauge condition. Moreover, the condition implies $\partial^{\gamma} \tilde{h}_{\alpha \gamma}^{\prime}=\partial_{\alpha} \tilde{f}$, with $\tilde{f}$ an arbitrary scalar function, indicating the fulfillment of just three independent restrictions on $\tilde{h}_{\alpha \beta}^{\prime}$. At the quantum level, one should add in LA the respective gauge fixing Lagrangian

$L_{F}=\alpha\left(\partial_{\alpha} \partial^{\gamma} h_{\beta \gamma}^{\prime}-\partial_{\beta} \partial^{\gamma} h_{\alpha \gamma}^{\prime}\right)^{2}$,

with $\alpha$ a dimensionless gauge parameter. At $\alpha \rightarrow \infty, L_{F}$ ensures the classical restriction (61). ${ }^{12}$ The gauge fixing Lagrangian additionally eliminates out of $L_{G}$ in LA three d.o.f.'s leaving seven independent ones, compared to ten under no-Diff and six under GDiff (realized at $\tilde{\varepsilon}_{x}=\tilde{\varepsilon}_{s}=0$ ). The three further gravity components, the vector ones, will appear only beyond LA. But having no quadratic propagator they will just modify the higher-order vertices by means of the contact interactions. ${ }^{13}$

While the most general natural TDiff case with $\tilde{\varepsilon}_{x} \neq 0$ deserves special consideration, let for simplicity $\tilde{\varepsilon}_{x}=0$, resulting in $\tilde{\varepsilon}_{s}=\varepsilon_{1}$. This implies one more restriction, $\varepsilon_{3}-$ $2 \varepsilon_{4}+\varepsilon_{5}=0$, on the Lagrangian parameters. However, not increasing the symmetry such a restriction is not natural in the 't Hooft's sense. In the minimal case, one gets

$L_{G}=\left(1+\varepsilon_{t}\right) L_{g}+\frac{1}{2}\left(\partial_{\alpha} \varsigma\right)^{2}-\frac{1}{2} M_{s}^{2} \varsigma^{2}$,

where we have introduced a (dimensionful) physical systolon field $\zeta \equiv \kappa_{s} h^{\prime} / 2, \kappa_{s} \equiv \varepsilon_{1}^{1 / 2} \kappa_{g}\left(\kappa_{g}=1\right)$, with a physical mass $M_{s} \equiv m_{s} \kappa_{g} / \kappa_{s}=m_{s} / \varepsilon_{1}^{1 / 2}$. Such a particular case presents a consistent quantum field theory, unitary and free of ghosts [3-5]. It describes the massless two-component transverse-tensor graviton and its massive scalar counterpart. Neglecting $m_{s}$, one can estimate from the

\footnotetext{
${ }^{11}$ Except if TDiff is broken dynamically back to the no-Diff case, with $\varepsilon_{v} \neq 0$ and $m_{t} \neq 0$ reappearing due to the quantum effects.

12 For a higher-derivative Lorentz-symmetric gauge, cf. [6]. Alternatively, to eliminate just three vector components, not touching the scalar one, there could to used a Lorentz-non-symmetric gauge. Due to the residual gauge invariance this should not violate the Lorentz symmetry.

13 Such an extra linear gauge is an artifact of LA with TDiff. In a general case, with all the ten d.o.f.'s in play, this gauge should be abandoned.
} 
anomalous asymptotic rotation velocities in galaxies, $v_{\infty}$, that $\varepsilon_{1}^{1 / 2} \sim v_{\infty} / c \sim 10^{-3}[9,10]$. It is seen that the limit $\varepsilon_{1} \rightarrow 0$ at a finite mass parameter $m_{s}$ would correspond to a non-propagating heavy systolon $\zeta$, with its physical mass $M_{s} \rightarrow \infty$. Thus to account for $m_{s} \neq 0$ could be of importance.

Altogether, the minimal version of QMG given by the two independent parameters $\varepsilon_{1}>0$ and $m_{s} \neq 0$, in principle, suffices to encompass both the gravity DM and DE. The account for $\varepsilon_{3} \neq 0$, resulting, in particular, in the kinetic mixing $\tilde{\varepsilon}_{x}=2 \varepsilon_{3}$, would extend the range of the phenomenological possibilities. Though the tensor gravity in LA remains the same as for GR (up to the overall normalization), the additional account for $\varepsilon_{4}, \varepsilon_{5} \neq 0$ in the different combinations (under $\varepsilon_{2}=-\varepsilon_{4}$ ) would modify the tensor gravity in the full nonlinear theory beyond GR, extending thus the observational possibilities for DS even further. Finally, the assumption for $\varepsilon_{v} \neq 0\left(\varepsilon_{2} \neq-\varepsilon_{4}\right)$ would ruin the residual Diff invariance in LA up to the no-Diff case (being problematic, as was mentioned). In this case, the number of propagating d.o.f.'s in LA equals ten, the vector part of them being ghosts. ${ }^{14}$

\section{Conclusion}

The consideration given shows that the quartet-metric (QM) GR (or, otherwise, QMG) may well serve as the theory of the unified gravitational DM and DE (the gravity DS). Under the natural (in the 't Hooft's sense) restriction on the parameters, the theory in LA, being unitary and free of ghosts, as well as the classical instabilities, consistently comprises the massless tensor graviton and its massive scalar counterpart, the systolon, as the DS particle. The sufficient abundance of the free parameters in the full nonlinear theory and the partial decoupling of the latter from its WF limit noticeably extend the prospects for the manifestations of the gravity DS in the various phenomena at the drastically different scales. Further theoretical study of the theory, as well as its observational verification/limitation, is urgent. Accounting as the effective field theory of gravity beyond GR for the influence of the vacuum, QM GR (under confirmation) could eventually pave the proper way toward a (more) fundamental theory of gravity and space-time.
Open Access This article is distributed under the terms of the Creative Commons Attribution 4.0 International License (http://creativecomm ons.org/licenses/by/4.0/), which permits unrestricted use, distribution, and reproduction in any medium, provided you give appropriate credit to the original author(s) and the source, provide a link to the Creative Commons license, and indicate if changes were made.

Funded by $\mathrm{SCOAP}^{3}$.

\section{References}

1. S. Capozziello, M. De Laurentis, Extended theories of gravity. Phys. Rep. 509, 167 (2011). arXiv:1108.6266 [gr-qc]

2. J.J. van der Bij, H. van Dam, Y.J. Ng, The exchange of massless spin-two particles. Physica 116A, 307 (1962)

3. W. Buchmüller, N. Dragon, Dilatons in flat and curved space-time. Nucl. Phys. B 321, 207 (1989)

4. N. Dragon, M. Creutzer, Quantization of restricted gravity. Z. Phys. C 41, 485 (1988)

5. M. Creutzer, Gauge theory of volume preserving diffeomorphisms. Class. Quant. Grav. 7, 1303 (1990)

6. E. Alvarez, D. Blas, J. Garriga, E. Verdaguer, Transverse Fiertz-Pauli symmetry. Nucl. Phys. B 756, 148 (2006). arXiv:hep-th/0606019

7. Y.F. Pirogov, General covariance violation and the gravitational dark matter. Scalar graviton. Yad. Fiz. 691374 (2006) [Phys. Atom. Nucl. 691338 (2006)]. arXiv:gr-qc/0505031

8. Y.F. Pirogov, Violating general covariance. arXiv:gr-qc/0609103

9. Y.F. Pirogov, Graviscalar dark matter and smooth galaxy halos. Mod. Phys. Lett. A 24, 3239 (2009). arXiv:0909.3311 [gr-qc]

10. Y.F. Pirogov, Unimodular bimode gravity and the coherent scalargraviton field as galaxy dark matter. Eur. Phys. J. C 72, 2017 (2012). arXiv:1111.1437 [gr-qc]

11. M.M. Anber, U. Aydemir, J.F. Donoghue, Breaking diffeomorphism invariance and tests for the emergence of gravity. Phys. Rev. D 81, 084059 (2010). arXiv:0911.4123 [gr-qc]

12. Y.F. Pirogov, Emergent gravity, violated relativity and dark matter. J. Mod. Phys. 5, 1418 (2014). arXiv:1404.4586 [gr-qc]

13. J.L. Anderson, D.R. Finkelstein, Cosmological constant and fundamental length. Am. J. Phys. 39, 901 (1971)

14. E.I. Guendelman, A.B. Kaganovich, The principle of nongravitating vacuum energy and some of its consequences. Phys. Rev. D 53, 7020 (1996). arXiv:gr-qc/9605026

15. G. 't Hooft, Unitarity in the Brout-Englert-Higgs mechanism for gravity. arXiv:0708.3184

16. A.H. Chamseddine, V. Muchanov, Higgs for graviton: simple and elegant solution. JHEP 1008, 011 (2010). arXiv:1002.3877 [hepth]

17. I. Oda, Higgs mechanism for gravitons. Mod. Phys. Lett. A 25, 2411 (2010). arXiv:1003.1437 [hep-th]

\footnotetext{
$\overline{14}$ On the contrary, if only $\varepsilon_{t} \neq 0$, then the residual gauge symmetry in LA naturally increases up to GDiff, leaving just six propagating d.o.f.'s, similarly to GR (though under a modified full nonlinear theory).
} 